Research Paper

\title{
Cardiovascular Effect of Dorsal Periaqueductal Gray During Lipopolysaccharide-induced Hypotension
}

\author{
Iraj Mirzaii-Dizgah $^{1^{*}}$ (D), Mohammad Naser Shafei ${ }^{2}$, Reza Mohebbati $^{1^{*}}$, Vida Alikhani ${ }^{3}$
}

1. Department of Physiology, School of Medicine, AJA University of Medical Sciences, Tehran, Iran

2. Neurogenic Inflammation Research Center, Mashhad University of Medical Sciences, Mashhad, Iran

3. Department of Physiology, Faculty of Medicine, Mashhad University of Medical Sciences, Mashhad, Iran

\begin{tabular}{|c|c|}
\hline $\begin{array}{l}\text { Use your device to scan } \\
\text { and read the article online }\end{array}$ & ditation Mirzaii-Dizgah, I., Shafei M. N., Mohebbati, R., Alikhani, V. (2022). Cardiovascular Effect of Dorsal Periaque- \\
\hline 口ificis & $\begin{array}{l}\text { ductal Gray During Lipopolysaccharide-induced Hypotension. Basic and Clinical Neuroscience, 13(2), 175-184. http://dx.doi. } \\
\text { org/10.32598/bcn.2022.2830.1 }\end{array}$ \\
\hline 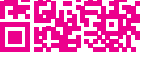 & del $h t$ ttp://dx.doi.org/10.32598/ben.2022.2830.1 \\
\hline
\end{tabular}

\section{(i) (8)}

Article info:

Received: 21 Sep 2019

First Revision: 20 Jun 2020

Accepted: 25 Aug 2020

Available Online: 01 Mar 2022

Keywords:

Dorsal periaqueductal gray,

Lidocaine, Lipopolysaccharide,

Blood pressure

\begin{abstract}
ABS T RACT
Introduction: The central mechanism related to the cardiovascular response to lipopolysaccharide (LPS)-induced hypotension is not entirely known, but it is suggested that numerous brain areas such as dorsal periaqueductal gray (dPAG) are involved in this process. In the current work, the cardiovascular effect of the dPAG during LPS-induced hypotension is investigated.

Methods: The study animals (rats) were divided into four groups: control (saline microinjected into dPAG), lidocaine 2\%, LPS (intravenously injected), and lidocaine + LPS. Catheterization of the femoral artery and vein was performed to record blood pressure and LPS injection, respectively. Saline and lidocaine were microinjected into the dPAG nucleus then the LPS injection was performed. The changes $(\Delta)$ in systolic blood pressure (SBP), mean arterial pressure (MAP), and heart rate (HR) were measured and compared with those of the control and LPS groups.

Results: LPS significantly declined $\triangle \mathrm{MAP}$ and $\triangle \mathrm{SBP}(\mathrm{P}<0.05)$ but did not change the $\Delta \mathrm{HR}$ compared to the control. Lidocaine did not significantly affect basic $\triangle \mathrm{SBP}, \triangle \mathrm{MAP}$, and $\triangle \mathrm{HR}$ compared to the control. Injection of lidocaine before LPS significantly attenuated the reduction of $\triangle \mathrm{SBP}$ and $\triangle \mathrm{MAP}$ evoked by LPS $(\mathrm{P}<0.05)$.

Conclusion: Our data showed that blockade of the dPAG by lidocaine significantly ameliorates the hypotension induced by LPS. this finding confirms the involvement of the dPAG in cardiovascular regulation during LPS-induced hypotension.
\end{abstract}

\footnotetext{
* Corresponding Author:

Reza Mohebbati, PhD.

Address: Department of Physiology, School of Medicine, AJA University of Medical Sciences, Tehran, Iran

Tel: +98 (915) 8081645

E-mail:mohebbatir@gmail.com ; mohebbatir931@mums.ac.ir
} 


\section{Highlights}

- Inactivation of the dPAG by lidocaine significantly ameliorates cardiovascular responses in hypotensive rats.

- LPS significantly lowers blood pressure and does not affect the heart rate.

\section{Plain Language Summary}

The mechanism of hypotension induced by endotoxin is not yet clear. However, it is often attributed to the direct effect of lipopolysaccharide (LPS) as a component of the outer wall of Gram-negative bacteria and other vascular mediators, including tumor necrosis factor (TNF) and nitric oxide (NO). One possibility is that the initial drop in LPSinduced arterial hypertension is mediated by a central mechanism. The ventral region of the transcranial gray matter is involved in lowering blood pressure, and the dorsal region is involved in increasing blood pressure. The dorsolateral region of the transcranial gray matter (dlPAG) also causes tachycardia, vasodilation in muscles, and tachypnea. dlPAG contains cells that produce $\mathrm{NO}$ and serotonin $(5 \mathrm{HT})$ and $5 \mathrm{HT} 1$ and $5 \mathrm{HT} 2$ receptors, which may play a role in hypotension due to stimulation of this region. LPS ( $1 \mathrm{mg} / \mathrm{kg}$ or higher IV) typically elicits a biphasic hypotensive response in rats. The first stage of this response begins immediately after LPS injection. The second phase begins about 1 hour after LPS injection. Thus, endotoxic hypertension begins through a central mechanism and further suggests that hypotension may play a critical role in developing fatal hypotension, representing the second stage of septic shock. Although dIPAG is an important site for regulating cardiovascular responses, its role in hypotension induced by LPS has not been investigated. We investigated the role of this nucleus in cardiovascular changes after LPS injection.

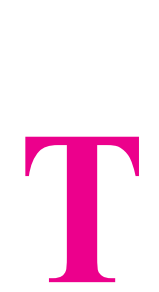

\section{Introduction}

he periaqueductal gray (PAG) region is located in the midbrain and plays a vital role in several biological functions, including defense reactions, fight and flight, pain, anxiety, reproduction, and cardiovascular and respiratory functions (Dampney, 1994; Farkas, Jansen, \& Loewy, 1998; Schenberg, Póvoa, Costa, Caldellas, Tufik, \& Bittencourt, 2005). From an anatomical point of view, PAG is divided into four different functional areas: lateral (lPAG), dorsolateral (dLPAG), dorsomedial (dMPAG), and ventrolateral (vLPAG) (Deolindo, Pelosi, Tavares, \& Corrêa, 2008; Schenberg et al., 2005). Based on the above classification, the defense responses and those related to hatred, hypertension, and tachycardia are related to the $\mathrm{MMPAG}$, and ALPAG, also called dorsal PAG. At the same time, calm behavioral responses, opioid-dependent analgesia, hypotension, bradycardia, and reward responses are attributed to the vLPAG region (Schenberg et al., 2005).

Lipopolysaccharide (LPS) is a potent bactericidal endotoxin that can cause fever, tachycardia, septic shock, and death following renal and pulmonary defects at high concentrations. Nevertheless, at a relatively low concentration, it acts as an active immune regulator and induces non-specific resistance against both bacterial and viral contamination (Wang \& Quinn, 2010).
Systemic infusion of LPS leads to a major challenge in the immune system, i.e., increasing various proinflammatory cytokines in the brain. Also, LPS systemic injection resulted in the production of intercellular binding molecules (ICAM-1), vascular connective tissue molecules (VCAM), and damage to the blood-brain barrier, resulting in granulocyte entry and initiation of immune responses (Hang et al., 2004). Although the arterial blood pressure reduction after LPS is mediated directly by vasodilation or evoking cytokine release, previous studies showed that LPS initially reduces arterial pressure through one or several central mechanism(s) such as vagal activation of the hypothalamic preoptic area (POA) (Millington, Yilmaz, \& Feleder, 2016). Therefore, we decided to investigate the cardiovascular effect of the dPAG during LPS-induced hypotension.

\section{Materials and Methods}

\section{Drug and grouping}

The study materials (urethane and lidocaine) were provided (Sigma, USA) and dissolved in saline.

The study rats were randomly divided into 4 groups as follows ( $\mathrm{n}=5$ for each group):

Control group: received microinjection of saline into the dPAG; 
Lidocaine group: received microinjection of lidocaine $2 \%$ into the dPAG;

LPS group: received an injection of the LPS intravenously (1 mg/kg, IV);

Lidocaine+LPS group: received microinjection of lidocaine into the dPAG before injection of LPS.

\section{Study Protocol}

Twenty male Wistar rats $(250 \pm 20 \mathrm{~g})$ were provided from the animal house of AJA University of Medical Sciences and kept in the room under physiological and standard conditions. The study protocol was approved by the Bioethics Committee of AJA University of Medical Sciences (Code: IR.AJAUMS.REC.1397.080).

Urethane $(1.5 \mathrm{~g} / \mathrm{kg}$, IP) was used for anesthesia. The femoral vein and artery were exposed and cannulated with a blue angiocath filled with heparinized saline (50 $\mathrm{u} / \mathrm{mL}$ ). Angiocath was connected to the pressure transducer and systolic blood pressure (SBP), mean arterial pressure (MAP), and heart rate (HR) were recorded throughout the trial period by a power lab system (Shafei \& Nasimi, 2011).

For microinjection of lidocaine, the rat was placed in a stereotaxic apparatus. The scalp was cut, the skull was leveled between bregma and lambda, and a tiny hole was drilled in the skull. The stereotaxic coordinates of the dPAG were AP: $7.5 \mathrm{~mm}, \mathrm{~L}: 0.7 \mathrm{~mm}$, and $\mathrm{H}: 4.8 \mathrm{~mm}$, based on the Paxinos book (Paxinos \& Franklin, 2004). Lidocaine 2\% (150 nl) (Yilmaz, Millington, \& Feleder, 2008) was microinjected into the dPAG nucleus by a single-barreled $40-\mu \mathrm{m}$ internal diameter micropipette. The pipette was connected to a manual microinjector (Harvard) via a PE-10 tube and carefully introduced into the $\mathrm{dPAG}$, and $150 \mathrm{nl}$ of agents was injected for about $30 \mathrm{~s}$ (Shafei, Nikyar, Hosseini, Niazmand, \& Paseban, 2017). Firstly, the lidocaine was injected, and then after $3 \mathrm{~min}$, the LPS was injected intravenously, and cardiovascular responses were evaluated. In the end, the animal brains were removed and located in formalin for verification of the injection sites under a light microscope.

\section{Data analysis}

The changes $(\Delta)$ in SBP, DBP, MAP, and HR were calculated and expressed as mean \pm SEM. In all groups for evaluation, trends of responses, $\triangle \mathrm{SBP}, \triangle \mathrm{DBP}, \triangle \mathrm{MAP}$, and $\triangle \mathrm{HR}$ during hypotension several times were calculated and compared with changes in other groups (repeated measures ANOVA). In addition, peak changes of $\triangle \mathrm{SBP}, \triangle \mathrm{DBP}, \triangle \mathrm{MAP}$, and $\triangle \mathrm{HR}$ of each group were separately provided and compared with peak changes of other groups (1-way ANOVA post hoc Tuckey). P values less than 0.05 were used to indicate significance.

\section{Results}

Saline was microinjected into the $\mathrm{dPAG}$, and cardiovascular responses were evaluated before and after administration in the normal condition. Microinjection

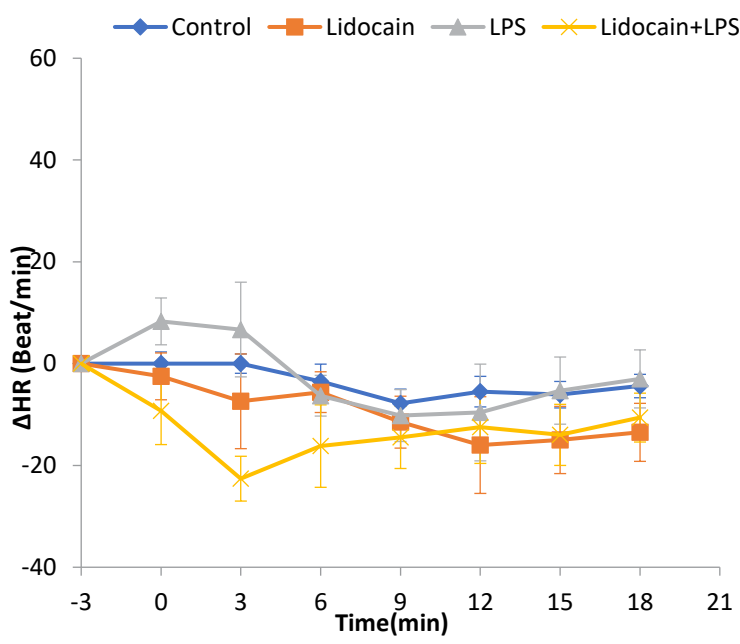

NEUR:SCIENCE

Figure 1. Time Course Changes of Heart Rate $(\Delta \mathrm{HR})$ After Microinjection of the Lipopolysaccharide (LPS) and Lidocaine Into the Dorsal Periaqueductal Gray (dPAG)

The data are expressed as Mean \pm SEM $(n=5)$. 


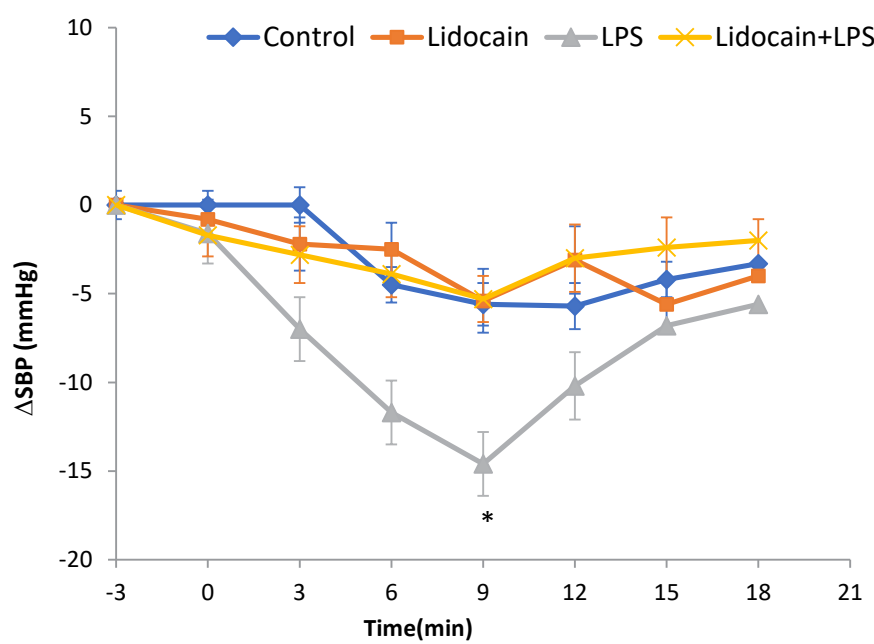

NEUR OSIENCE

Figure 2. Time Course Changes of Systolic Blood Pressure ( $\triangle \mathrm{SBP})$ After Microinjection of the Lipopolysaccharide (LPS) and Lidocaine Into the Dorsal Periaqueductal Gray (dPAG)

The data are expressed as mean $\pm \operatorname{SEM}(n=5) .{ }^{*} \mathrm{P}<0.05$ compared to the control.

of saline did not significantly change these parameters compared to pre-injection values.

To examine the role of the dPAG on cardiovascular responses, lidocaine was microinjected into the dPAG. The data showed that lidocaine alone did not significantly affect the cardiovascular parameters.
As indicated, $\triangle \mathrm{MAP}, \triangle \mathrm{SBP}$, and $\triangle \mathrm{DBP}$ in the LPS group were significantly reduced compared with the saline group $(\mathrm{P}<0.05)$.

Lidocaine injection improved the MAP, SBP, and DBP in the rats treated with LPS. The $\triangle \mathrm{MAP}, \triangle \mathrm{SBP}$, and $\triangle \mathrm{DBP}$ were slowly returned and stabilized lower than the basal state within $12 \mathrm{~min}$. In the LPS + lidocaine group, pre-treatment with the lidocaine significantly

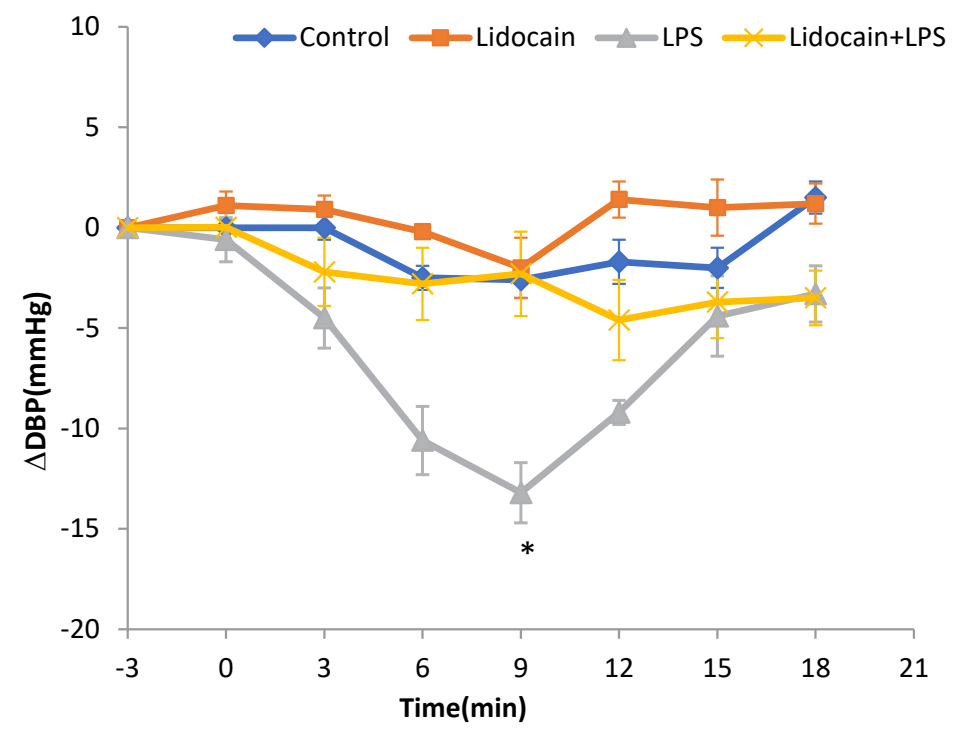

NEUR SCIENCE

Figure 3. Time Course Changes of Diastolic Blood Pressure ( $\triangle \mathrm{DBP})$ After Microinjection of the Lipopolysaccharide (LPS) and Lidocaine Into the Dorsal Periaqueductal Gray (dPAG)

The data are expressed as Mean \pm SEM; $n=5$. ${ }^{*}<0.05$ compared to the control. 


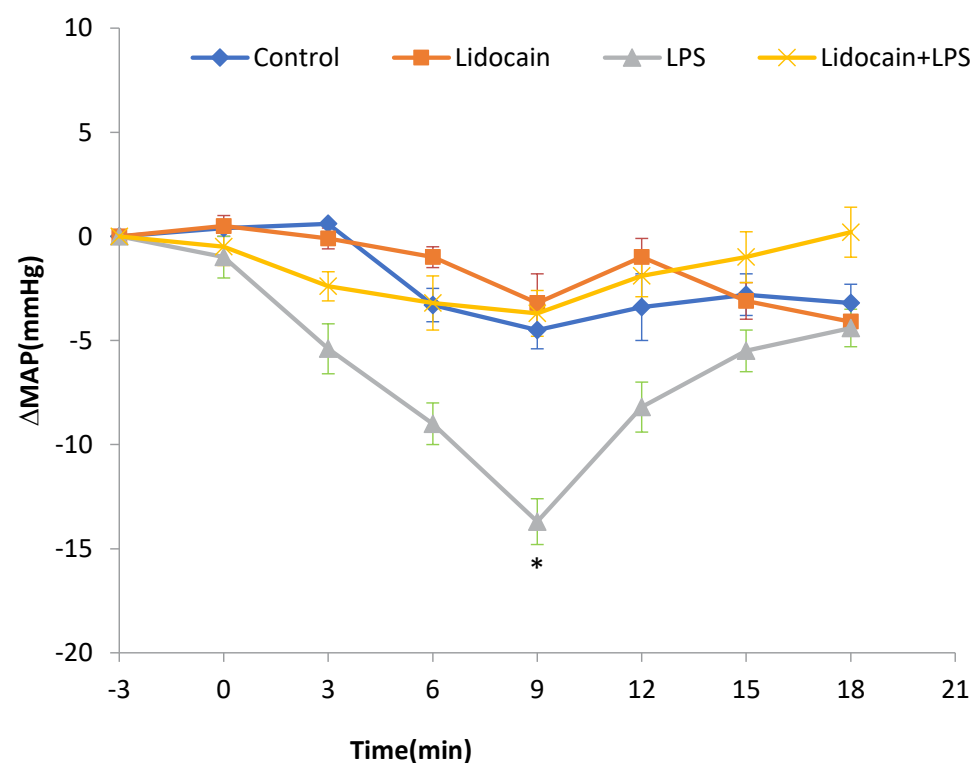

NEUROSCIENCE

Figure 4. Time Course Changes of Mean Arterial Pressure ( $\triangle \mathrm{MAP})$ After Microinjection of the Lipopolysaccharide (LPS) and Lidocaine Into the Dorsal Periaqueductal Gray (dPAG)

The data are expressed as Mean \pm SEM $(n=5)$. ${ }^{*}<<0.05$ compared to the control.

attenuated hypotension (decreased $\triangle \mathrm{MAP}, \triangle \mathrm{SBP}$, and $\triangle \mathrm{DBP}$ with respect to the LPS group $(\mathrm{P}<0.05)$. The $\Delta H R$ in the treated groups did not observe any significant change (Figures 1, 2, 3, 4).

The peak changes of the $\triangle \mathrm{SBP}(\mathrm{P}<0.05), \triangle \mathrm{DBP}$ $(\mathrm{P}<0.01)$, and $\triangle \mathrm{MAP}(\mathrm{P}<0.01)$ in the LPS group signif- icantly declined in comparison with the control while these peak changes in the lidocaine and LPS + lidocaine $(\mathrm{P}<0.05$ to $\mathrm{P}<0.01)$ groups significantly decreased compared to the LPS group. The peak changes of the HR did not significantly differ between groups (Figures 5, 6, 7, $8)$. The recorded samples of the cardiovascular parameters are shown in Figures 9 and 10.

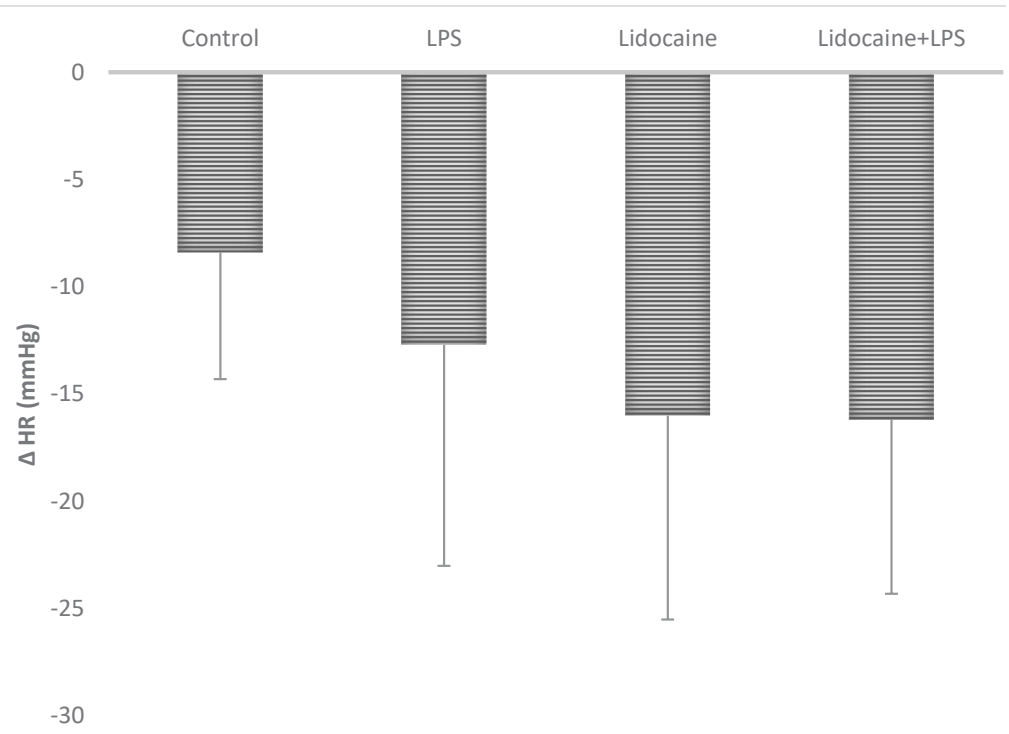

Figure 5. Peak Changes of Heart Rate $(\Delta \mathrm{HR})$ After Microinjection of the Lipopolysaccharide (LPS) and Lidocaine Into the Dorsal Periaqueductal Gray (dPAG) in Rats

The data are expressed as Mean \pm SEM $(n=5)$. 


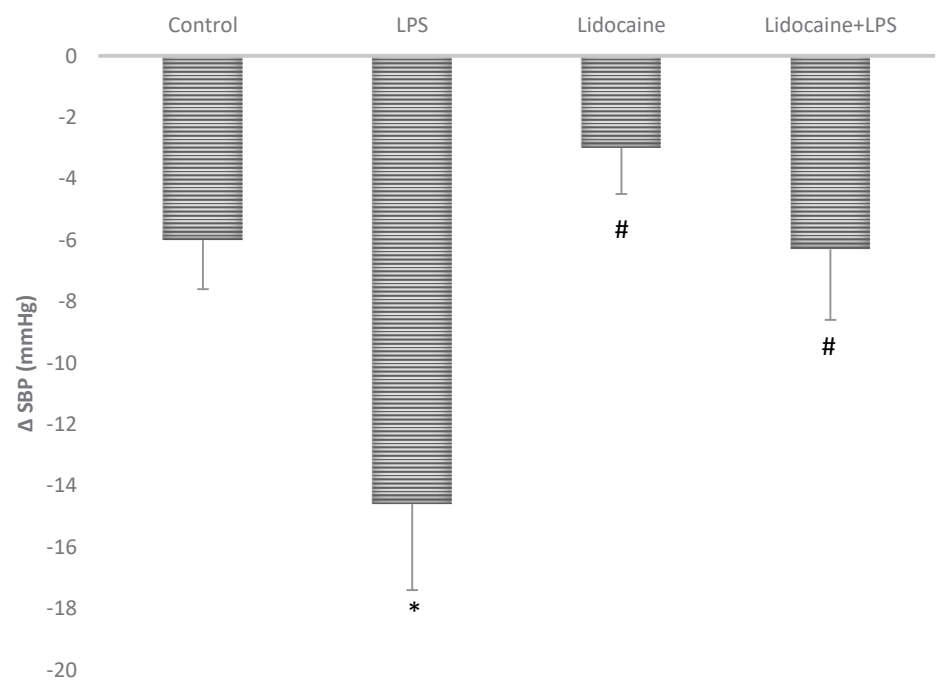

NEUR OSCIENCE

Figure 6. Peak Changes of Systolic Blood Pressure ( $\triangle \mathrm{SBP}$ ) After Microinjection of the Lipopolysaccharide (LPS) and Lidocaine Into the Dorsal Periaqueductal Gray (dPAG) in Rats

The data are expressed as Mean \pm SEM $(n=5)$. ${ }^{*}<0.05$ compared to the control, $\# \mathrm{P}<0.05$ compared to the LPS group.

\section{Discussion}

The study's objective was to explore whether the dPAG is involved in preventing LPS-induced hypotension. The role of the dPAG nucleus was investigated in both normotensive and hypotensive conditions. Our findings showed that in normotensive conditions, inactivation of the dPAG area did not have an effect on the cardiovascular responses. Although the dPAG nucleus did not affect the cardiovascular response in basal conditions, inactiva- tion of this nucleus by lidocaine attenuated hypotension induced by LPS. Probably, there are some neurons in this nucleus, such as glutamatergic or adrenergic neurons, that lidocaine, via inhibition of the $\mathrm{Na}+$ channels on these neurons, causes hyperpolarization and induces the hypotension through related vasomotor nuclei directly or indirectly (Sepehri \& Shafiei, 2006). With regard to our findings, Fisk et al. indicated that ablation of the dPAG with local injection of $\mathrm{CoCl} 2$ (a synaptic blocker) did not affect the blood pressure and HR (Fisk \& Wyss,

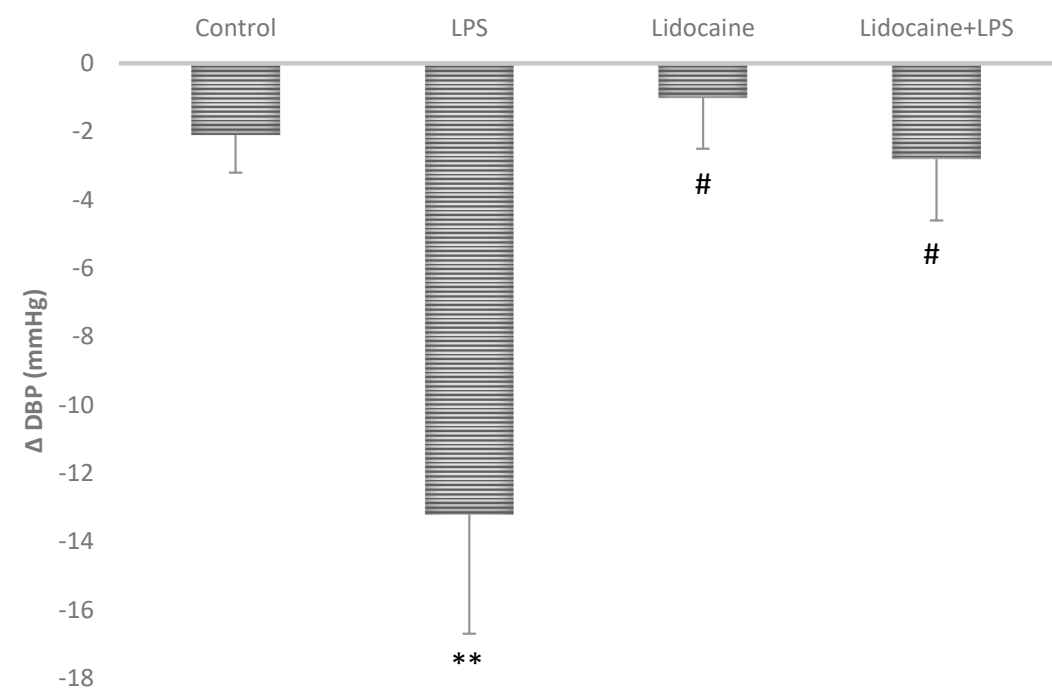

NEUR SCIENCE

Figure 7. Peak Changes of Diastolic Blood Pressure $(\triangle \mathrm{DBP})$ After Microinjection of the Lipopolysaccharide (LPS) and Lidocaine Into the Dorsal Periaqueductal Gray (dPAG) in Rats

The data are expressed as mean $\pm \mathrm{SEM}(\mathrm{n}=5)$. ${ }^{* *} \mathrm{P}<0.01$ compared to the control, $\# \mathrm{P}<0.05$ compared to the LPS group. 


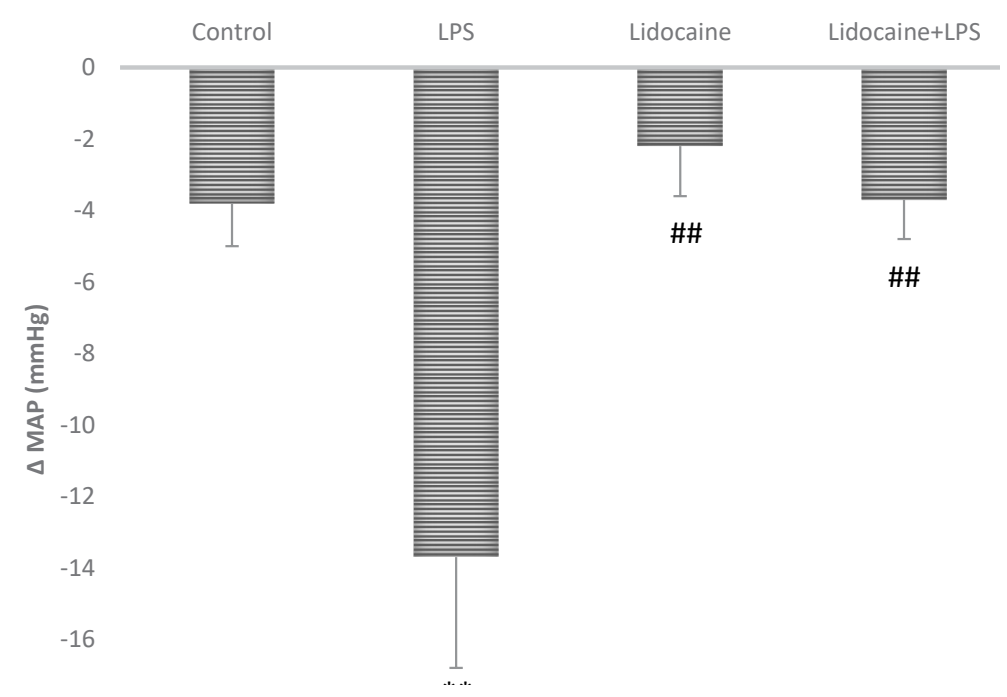

NEUR:SCIENCE

Figure 8. Peak Changes of Mean Arterial Pressure ( $\mathrm{MAP}$ ) After Microinjection of the Lipopolysaccharide (LPS) and Lidocaine Into the Dorsal Periaqueductal Gray (dPAG) in Rats

The data are expressed as Mean \pm SEM $(n=5) . * * P<0.01$ compared to the control, $\# \# P<0.05$ compared to the LPS group.

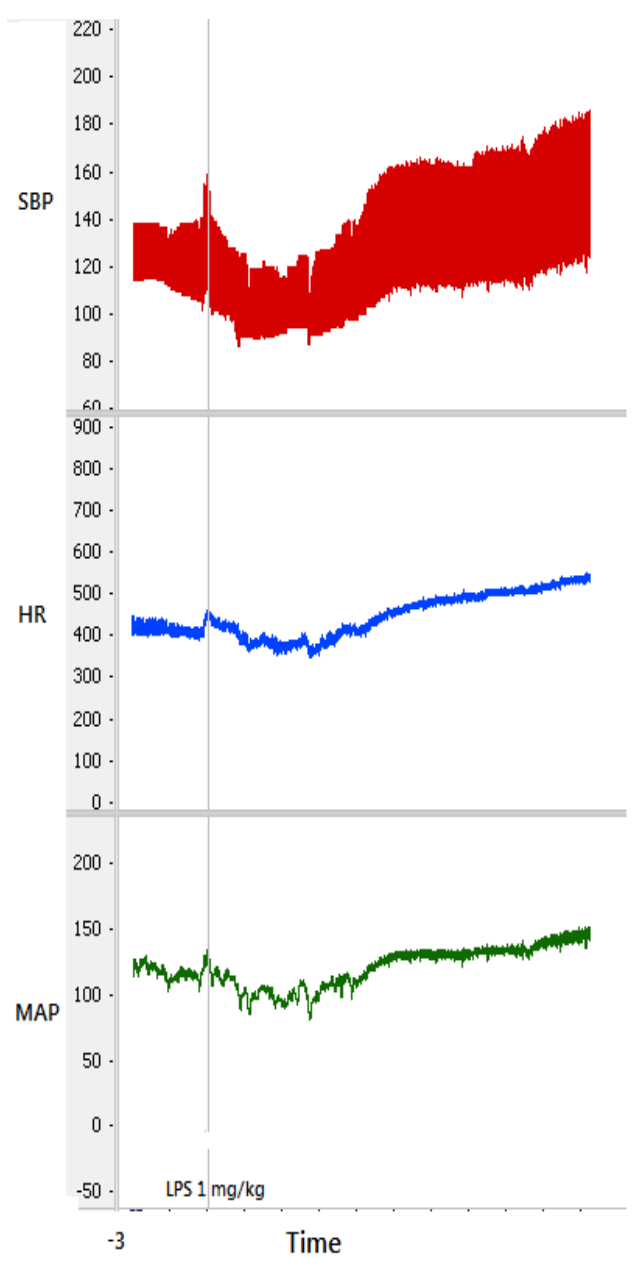

NEUR SCIENCE

Figure 9. The Samples of Blood Pressure (BP), Mean Arterial Pressure (MAP), and Heart Rate (HR) Recorded After Lipopolysaccharide (LPS) Injection

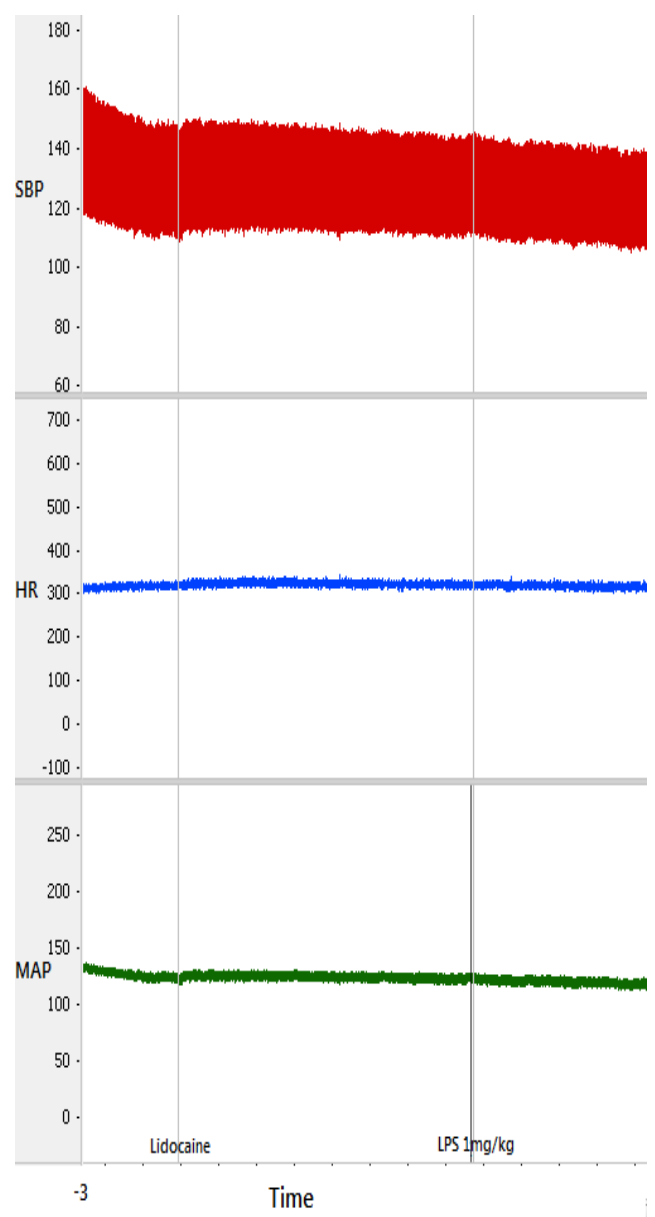

NEUR SCIENCE

Figure 10. The Samples of Blood Pressure (BP), Mean Arterial Pressure (MAP), and Heart Rate (HR) recorded After Lidocaine Microinjection Into the Dorsal Periaqueductal Gray (dPAG) and Lipopolysaccharide (LPS) Injection 


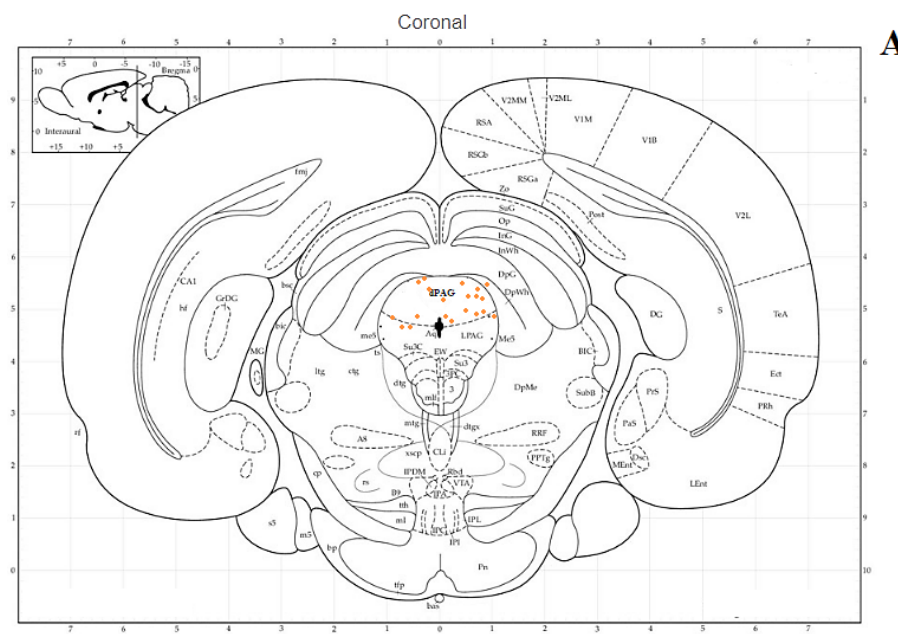

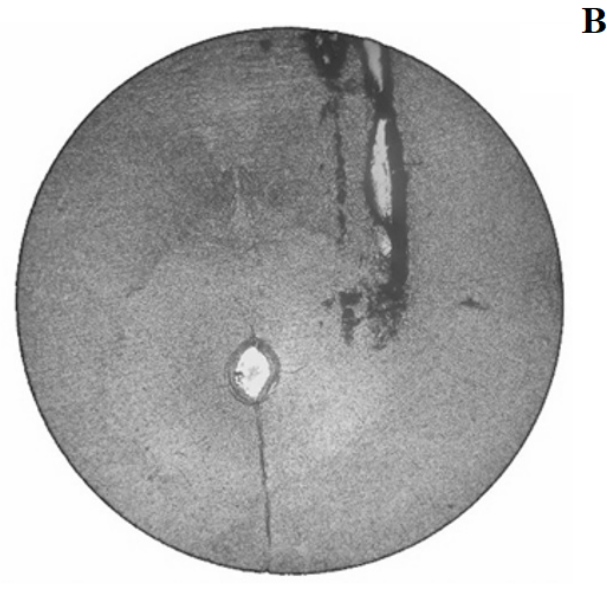

NEUR \$SIENCE

Figure 11. Coordinates of injection locations (red dot) adopted form the paxinos atlas (a) and a sample of brain section after microinjection of the drug into the dorsal periaqueductal gray (dPAG) (B)

2000). Also, Deolindo et al. showed that acetylcholine microinjection into the dPAG did not affect the MAP and HR in the normotensive rats (Deolindo, Pelosi, Busnardo, Resstel, \& Corrêa, 2011). However, Kubo et al. had shown that electrical stimulation of the dPAG resulted in enhancing blood pressure (Kubo, Hagiwara, Sekiya, \& Fukumori, 1999). However, in the current study, after inactivation of the $\mathrm{dPAG}$, we did not observe any significant reduction in the cardiovascular parameters. Based on this result, we suggest that in normal condition, this area does not have cardiovascular activity. However, in conditions such as hypotension, stress, or another stimulus, the neurons of this areas activate and precipitate in the regulation of cardiovascular responses.

Our results indicated that LPS decreased systemic blood pressure with no significant effect on HR. The results cover the previous works that indicate LPS-induced hypotension. It is suggested that the LPS after injection into the systemic bloodstream evokes the cytokines secretion, resulting in brief HR elevation. Then, LPS inserts into the central nervous system by destroying the blood-brain barrier and affecting neural pathways. This process is time-consumer. The mechanism(s) of the central effect of LPS on hypotension is not entirely known, but it is reported that LPS causes a decrease in the sympathetic outflow to brainstem sympathetic pathways and increases the cytokine levels such as TNF- $\alpha$, resulting in vasodilation (Koyama \& Manning, 1985; Millington et al., 2016). In the lidocaine+LPS group, after several minutes, the HR non-significantly increased, probably via excitation of the baroreflex regulatory mechanism.
Yilmaz et al. had shown that microinjection of the lidocaine or the alpha-adrenergic receptor antagonist into the hypothalamic preoptic area (POA) prevents the hypotension induced by LPS (Yilmaz et al., 2008). Based on Pelosi et al. study that there are noradrenergic receptors within the APAG, we suggest that LPS by an inhibitory effect on adrenergic receptors of the dPAG causes a depressor effect (Pelosi \& Corrêa, 2005). In addition, numerous studies have indicated that activation of the POA neurons lowers arterial pressure through a descending pathway from the POA to the PAG and midline raphe nuclei (Behbehani \& Gomez, 1996; Jiang \& Behbehani, 2001).

Therefore, we suggest an adrenergic pathway between the POA and APAG that is involved in cardiovascular regulation and LPS via inhibition of the adrenergic as well as other excitatory neurons that cause hypotension. Lidocaine, by inactivation of this pathway or their pathways, could prevent the LPS-induced hypotension. In addition, there are projections from the dPAG to vasomotor areas, including caudal ventrolateral medulla (CVLM), nucleus tractus solitarius (NTS), and rostral ventrolateral medulla (RVLM), and we suggest that LPS by affecting these projections reduce blood pressure. However, we need more studies to confirm these opinions.

The current study's limitations are a lack of additional cardiovascular parameters evaluation such as ECG and no histochemical study for LPS tracing in the brain. We encourage dear researchers to complete the study by removing the above limitations.

In conclusion, this study was a first step that shows the dPAG area is involved in adjusting cardiovascular re- 
sponses during LPS-induced hypotension. We proposed further studies to evaluate the exact mechanisms and neural circuits during LPS-induced hypotension.

\section{Ethical Considerations}

\section{Compliance with ethical guidelines}

The study protocol was approved by the Bioethics Committee of AJA University of Medical Sciences (Code: IR.AJAUMS.REC.1397.080).

\section{Funding}

This study was funded by the Research Council of AJA University of Medical Sciences, Tehran, Iran.

\section{Authors' contributions}

Conceptualization: Mohammad Naser Shafei and Reza Mohebbati; Methodology: Reza Mohebbati and Iraj Mirzaii-Dizgah; Investigation and Writing - original draft: Reza Mohebbati and Mohammad Naser Shafei; Writing - review, editing, Supervision, and Funding acquisition: Iraj Mirzaii-Dizgah and Vida Alikhani; Resources: All authors.

\section{Conflict of interest}

The authors declared no conflict of interest.

\section{Acknowledgments}

The authors would like to thank the Research Council of AJA University of Medical Sciences for funding this study.

\section{References}

Behbehani, M. M., \& DaCosta Gomeza, T.M. (1996). Properties of a projection pathway from the medial preoptic nucleus to the midbrain periaqueductal gray of the rat and its role in the regulation of cardiovascular function. Brain Research, 740(1-2), 141-150. [DOI:10.1016/S0006-8993(96)00858-X] [PMID]

Dampney, R. A. (1994). Functional organization of central pathways regulating the cardiovascular system. Physiological Reviews, 74(2), 323-364. [DOI:10.1152/physrev.1994.74.2.323] [PMID]

Deolindo, M., Pelosi, G. G., Tavares, R. F., \& Corrêa, F. M. A. (2008). The ventrolateral periaqueductal gray is involved in the cardiovascular response evoked by l-glutamate microinjection into the lateral hypothalamus of anesthetized rats. Neuroscience Letters, 430(2), 124-129. [DOI:10.1016/j.neulet.2007.10.028] [PMID]
Deolindo, M. V., Pelosi, G. G., Busnardo, C., Resstel, L. B. M. \& Corrêa, F. M. A. (2011). Cardiovascular effects of acetylcholine microinjection into the ventrolateral and dorsal periaqueductal gray of rats. Brain Research, 1371, 74-81. [DOI:10.1016/j. brainres.2010.11.051] [PMID]

Farkas, E., Jansen, A. S. P., \& Loewy, A. D. (1998). Periaqueductal gray matter input to cardiac-related sympathetic premotor neurons. Brain Research, 792(2), 179-192. [DOI:10.1016/S00068993(98)00029-8] [PMID]

Fisk, G. D., \& Wyss, J. M. (2000). Descending projections of infralimbic cortex that mediate stimulation-evoked changes in arterial pressure. Brain Research, 859(1), 83-95. [DOI:10.1016/ S0006-8993(00)01935-1] [PMID]

Hang, C.H., Shi, J. X., Tian, J., Li, J.S., Wu, W., \& Yin, H.X. (2004). Effect of systemic LPS injection on cortical NF-KB activity and inflammatory response following traumatic brain injury in rats. Brain Research, 1026(1), 23-32. [DOI:10.1016/j.brainres.2004.07.090] [PMID]

Jiang, M., \& Behbehani, M. M. (2001). Physiological characteristics of the projection pathway from the medial preoptic to the nucleus raphe magnus of the rat and its modulation by the periaqueductal gray. Pain, 94(2), 139-147. [DOI:10.1016/ S0304-3959(01)00348-7]

Koyama, S., \& Manning, J. W. (1985). Role of sympathetic nerve activity in endotoxin induced hypotension in cats. Cardiovascular Research, 19(1), 32-37. [DOI:10.1093/cvr/19.1.32] [PMID]

Kubo, T., Hagiwara, Y., Sekiya, D., \& Fukumori, R. (1999). Midbrain central gray is involved in mediation of cholinergic inputs to the rostral ventrolateral medulla of the rat. Brain Research Bulletin, 50(1), 41-46. [DOI:10.1016/S03619230(99)00088-X]

Millington, W. R., Yilmaz, M. S., \& Feleder, C. (2016). The initial fall in arterial pressure evoked by endotoxin is mediated by the ventrolateral periaqueductal gray. Clinical and Experimental Pharmacology and Physiology, 43(6), 612-615. [DOI:10.1111/1440-1681.12573] [PMID] [PMCID]

Paxinos, G., \& Franklin, K. B. J. (2004). The mouse brain in stereotaxic coordinates. Amesterdam: Elsevier Academic Press. https://www.google.com/books/edition/The Mouse 0mouse\%20brain\%20in\%\%20 coordinates $\& \mathrm{f}=$ false

Pelosi, G. G., \& Corrêa, F. M. A. (2005). Cardiovascular effects of noradrenaline microinjected into the dorsal periaqueductal gray area of unanaesthetized rats. European Journal of Neuroscience, 22(12), 3188-3194. [DOI:10.1111/j.14609568.2005.04511.x] [PMID]

Schenberg, L. C., Póvoa, R. M. F., Costa, A. L. P., Caldellas, A. V., Tufik, S., \& Bittencourt, A. S. (2005). Functional specializations within the tectum defense systems of the rat. Neuroscience EBiobehavioral Reviews, 29(8), 1279-1298. [DOI:10.1016/j neubiorev.2005.05.006] [PMID]

Sepehri, G. R., \& Shafeiee, M. N. (2006). Effect of cuneiformis nucleus inactivation by lidocaine Microinjection on the analgesic response of morphine in rats. Iranian Biomedical Journal, 10(1), 21-26. http://ibj.pasteur.ac.ir/article-1-347-en.pdf

Shafei, M. N., \& Nasimi, A. (2011). Effect of glutamate stimulation of the cuneiform nucleus on cardiovascular regula- 
tion in anesthetized rats: Role of the pontine Kolliker-Fuse nucleus. Brain Research, 1385, 135-143. [DOI:10.1016/j.brainres.2011.02.046] [PMID]

Shafei, M. N., Nikyar, T., Hosseini, M., Niazmand, S., \& Paseban, M. (2017). Cardiovascular effects of nitrergic system of the pedunculopontine tegmental nucleus in anesthetized rats. Iranian Journal of Basic Medical Sciences, 20(7), 776-782. [PMID] [PMCID]

Wang, X., \& Quinn, P. J. (2010). Lipopolysaccharide: Biosynthetic pathway and structure modification. Progress in Lipid Research, 49(2), 97-107. [DOI:10.1016/j.plipres.2009.06.002] [PMID]

Yilmaz, M. S., Millington, W. R., \& Feleder, C. (2008). The preoptic anterior hypothalamic area mediates initiation of the hypotensive response induced by LPS in male rats. Shock, 29(2), 232-237. [DOI:10.1097/shk.0b013e3180caac7e] [PMID] 